\title{
Chest pain after percutaneous coronary intervention in patients with stable angina
}

\author{
This article was published in the following Dove Press journal: \\ Clinical Interventions in Aging \\ 18 August 2016 \\ Number of times this article has been viewed
}

\section{Chao-Chien Chang ${ }^{1-3}$ \\ Yueh-Chung Chen ${ }^{4,5}$ \\ Eng-Thiam Ong' \\ Wei-Cheng Chen' \\ Chia-Hsiu Chang' \\ Kuan-Jen Chen' \\ Cheng-Wen Chiang'}

'Division of Cardiology, Department of Internal Medicine, Cathay General Hospital, Taipei, Taiwan, ROC; ${ }^{2}$ Graduate Institute of Medical Sciences, Taipei Medical University, Taipei, Taiwan, ROC; ${ }^{3}$ Department of Pharmacology, Taipei Medical University, Taipei, Taiwan, ROC; ${ }^{4}$ Division of Cardiology, Department of Internal Medicine, Taipei City Hospital Ren-Ai branch, Taipai, Taiwan, ROC; ${ }^{5}$ Graduate Institute of Medical Sciences, National Defense Medical Center, Taipei, Taiwan, ROC
Correspondence: Cheng-Wen Chiang Division of Cardiology, Department of Internal Medicine, Cathay General Hospital, 280, Section 4, Ren-Ai road, Taipei 106, Taiwan, ROC

Tel +8862 7082121 ext 3116

Fax +88629324969

Email cgh0576I@cgh.org.tw
Background: Percutaneous coronary intervention (PCI) has been widely used to treat acute coronary syndrome but is only recommended as an additional treatment to medical therapy and risk modification in patients with refractory or progressing angina. The number of PCI in this patient population is still increasing. Post-PCI chest pain (PPCP) is one of the common problems of PCI. Its presentation and causes in patients with stable angina are poorly understood.

Patients and methods: This study retrospectively collected clinical information of 167 patients who had stable angina and underwent elective PCI, including 70 patients with PPCP 24 hours after procedure and 97 patients without PPCP. The incidence and predictors of PPCP were analyzed.

Results: The incidence of PPCP was 41.9\% (70/167). Compared with non-PPCP patients, PPCP patients had more abnormal post-PCI electrocardiogram (ECG) changes (new Q-waves, STsegment shifts, or T-waves inversion) and serum cardiac troponin I (cTnI) elevation, more PCI vessels, and stent placement (all $P<0.05$ ). More PPCP patients required repeat revascularization than non-PPCP patients after PCI $(P=0.043)$. PPCP was correlated with abnormal post-PCI ECG changes $(P<0.0001)$, cTnI elevation $(P<0.0001)$, post-PCI serum level of $\mathrm{cTnI}(P<0.0001)$, number of stents placed $(P=0.009)$, and pre-PCI cTnI level $(P=0.049)$. The strongest predictors of PPCP were abnormal post-PCI ECG changes $(P<0.0001)$, post-PCI cTnI level $(P<0.0001)$, and $\mathrm{cTnI}$ elevation $(P<0.0001)$, followed by the number of stents placed $(P=0.048)$.

Conclusion: PPCP is common in patients with stable angina in our cohort. It is associated with abnormal ECG changes, cTnI elevation, and number of stents placed.

Keywords: coronary angiography, percutaneous coronary interventions, chest pain, complications, interventional cardiology, cardiac troponin I

\section{Introduction}

Percutaneous coronary intervention (PCI) has been widely used to treat obstructive coronary artery disease, including unstable angina, recurrent angina after coronary artery bypass grafting (CABG), and acute myocardial infarction (MI), contributing to the reduction of clinical symptoms and mortality rate. ${ }^{1-3}$ A total of 92,589 PCIs were performed from January to December 2013, which represented a rate of 1,444 PCI per million population in the UK. ${ }^{4}$ Although PCI is only recommended as an additional treatment to medical therapy and risk modification in patients with refractory or progressing angina because of the controversial reports about its benefit on stable angina, ${ }^{5-7}$ the numbers of elective PCI conducted in patients with stable angina still account for approximately $30 \%-40 \%$ of total PCI procedures in patients with coronary artery diseases. ${ }^{8,9}$ This procedure is becoming more prevalent with the advent of reliable noninvasive coronary imaging. 
One of the common problems following PCI is postPCI chest pain (PPCP). In the past, PPCP has been mainly studied in patients with acute coronary syndrome (ACS). Schüepp et $\mathrm{al}^{10}$ found that one-third of patients experienced chest pain in the early period after successful PCI, and Kini et $\mathrm{al}^{11}$ reported $35.8 \%$ postprocedure chest pain after coronary stenting. The mechanisms of PPCP have not been completely understood yet. PPCP can be caused by acute stent thrombosis, incomplete revascularization, restenosis, inappropriate vasoconstriction, stent-stretch, and progression of disease not involving the target lesion. ${ }^{10,12-14}$ Therefore, it is essential to identify the underlying mechanisms of PPCP and rule out critical conditions such as thrombosis and restenosis. It is also important to distinguish benign PPCP from critical PPCP to avoid unnecessary, expensive examinations and treatment. PPCP in patients with chronic stable angina is poorly understood. We therefore conducted the present study to investigate the incidence and predictors of PPCP in patients with stable angina to provide useful information for clinical management. These factors can also help identify patients with a low probability of new myocardial ischemia and avoid expensive and empirical treatment.

\section{Patients and methods}

\section{Patients}

All patients undergoing elective PCI for severe angina in our department between January 2006 and January 2010 were eligible for the retrospective study. Exclusion criteria included previous history of ACS, primary PCI for ACS with elevated creatine kinase (CK-MB) and cardiac troponin I (cTnI) before the procedure, cardiogenic shock and/ or arrhythmia after the procedure, complications of PCI such as bleeding and infection, and patients without complete data about the details of PCI, pre- and post-PCI electrocardiogram (ECG), and cTnI. The study was approved by the Ethics Committee of Cathay General Hospital and informed consent was waived for this retrospective study. Clinical trial registration number: CT099016.

\section{Study design}

Baseline demographics, medication use, routine laboratory results, and procedural details were obtained from medical records. PPCP was defined as varying degrees of typical chest pain after PCI. Stable and unstable angina and MI were defined according to the American College of Cardiology/ American Heart Association guidelines. ${ }^{15}$ Twelve-lead ECGs were recorded before intervention and immediately after intervention. New Q-waves, ST-segment shifts, or T-waves inversion were considered as ischemia. Blood was collected from each patient for measurement of serum cTnI before PCI and 24 hours after PCI. All cTnI samples were analyzed within 12 hours of specimen collection with an immunoassay analyzer (Immulite; Diagnostic Products Corporation, Los Angeles, CA, USA) using commercially available test materials. The normal range is $0-0.16 \mathrm{ng} / \mathrm{mL}$. cTnI elevation was defined if post-PCI cTnI level was higher than pre-PCI cTnI level and was $>0.16 \mathrm{ng} / \mathrm{mL}$. Patients were divided into the PPCP group and the non-PPCP group. The baseline characteristics, PCI treatment, ECG changes, serum cTnI levels, and short-term outcomes such as post-PCI MI, repeat revascularization, and death were compared between the two groups.

\section{$\mathrm{PCl}$ procedures}

A standard procedure of PCI was performed by femoral approach under local anesthesia. No rotablation and atherectomy device were used. Aspirin (100 mg; Bayer AG, Leverkusen, Germany) and clopidogrel (300 mg; Bristol-Myers Squibb, New York, NY, USA) were administrated to all patients 24 hours before the procedure, and heparin $(5,000 \mathrm{U})$ was administrated intravenously in some patients. All procedures were performed by a team of experienced interventional cardiologists led by the corresponding author.

\section{Statistical analysis}

All analysis was performed using SPSS 20.0 (IBM Corporation, Armonk, NY, USA). Categorical variables were expressed as numbers (\%) and compared using $\chi^{2}$ tests. Continuous variables were expressed as mean \pm standard error (SE) and compared using Student's $t$-test or Welch's $t$-test. Pearson or Spearman correlation and logistic regression were used to assess the association of PPCP and demographic, clinical, pre- and post-PCI ECG, and cTnI elevation. A $P$-value of $<0.05$ was considered statistically significant.

\section{Results \\ Study Population}

A total of 378 patients with severe angina underwent elective PCI in our department during the period from January 2006 to January 2010. After excluding patients with exclusion criteria, 167 patients were eligible for analysis. Among them, 123 patients complained of chest pain after PCI. However, the diagnosis of PPCP was excluded in 53 patients because their post-PCI cTnI levels were within normal range, and the abnormality of post-PCI ECG such as ST depression and the characteristics of chest pain were not much different from that before PCI. As a result, 70 patients $(41.9 \%)$ were finally included in the PPCP group, and 97 (58.1\%) patients were in the non-PPCP group (Figure 1). 


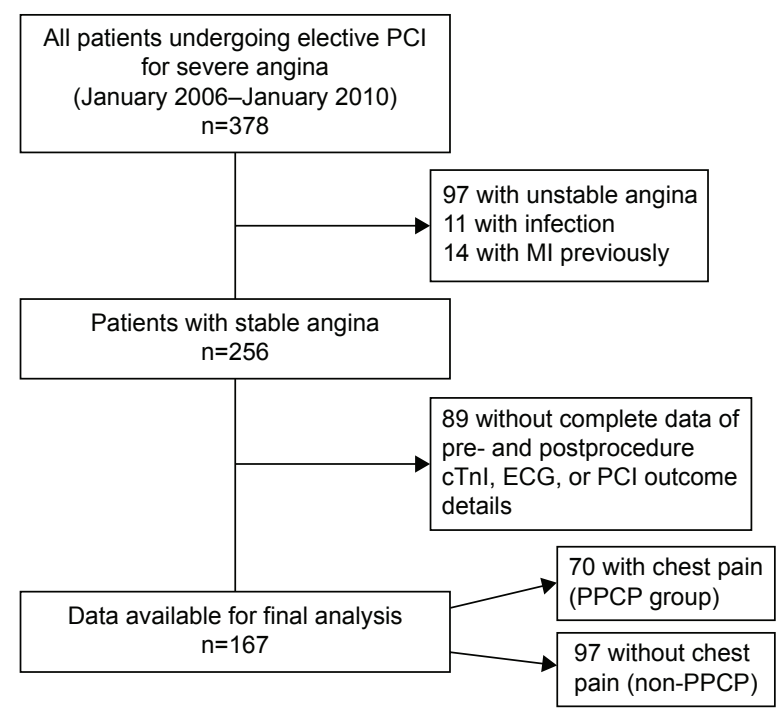

Figure I Flowchart for study eligibility.

Abbreviations: $\mathrm{PCl}$, percutaneous coronary intervention; $\mathrm{PPCP}$, post- $\mathrm{PCl}$ chest pain; cTnl, cardiac troponin I; MI, myocardial infarction.

\section{Baseline characteristics}

Baseline demographic and clinical characteristics for the study cohort are summarized in Table 1. Comparing the PPCP group with the non-PPCP group, the pre-PCI serum cTnI level was significantly higher $(P=0.040)$ despite the levels being within the normal range. No difference was found in age, sex, risk factors, previous history of surgical revascularization, medical treatment, and routine blood test results (all $P>0.05$ ). No PCI was performed in a chronic total occlusion (CTO) vessel.

\section{Percutaneous coronary intervention and short-term characteristics}

As shown in Table 2, compared with non-PPCP patients, PPCP patients had a higher proportion of new Q-waves, STsegment shifts, or T-waves inversion $(P<0.0001)$, and TnI elevation $(P<0.0001)$ following PCI, as well as a higher number of intervention vessels $(P=0.020)$ and stent placement $(P=0.007)$. The mean post-PCI serum level of cTnI $(P=0.007)$ was significantly higher in PPCP patients than in non-PPCP patients $(P=0.007)$. In total, $78(80.4 \%)$ non-PPCP patients and $62(88.8 \%)$ PPCP patients had stents implanted. No difference was found in the type of stent implanted (bare-metal stent or drug-eluting stent) between the two groups (all $P>0.05$ ). More patients in the PPCP group received repeat PCI treatment $(P=0.041)$, and two of them were finally diagnosed with MI with abnormal ECG and elevated cTnI levels ( $>50$ times the upper normal limit). The mortality rate is $3 \%(5 / 167)$ in total and is not significantly different between the two
Table I Baseline demographic and clinical characteristics in the PPCP versus non-PPCP groups

\begin{tabular}{|c|c|c|c|}
\hline & Non-PPCP & PPCP & $P$-value \\
\hline Number of patients & 97 & 70 & \\
\hline Age, years & $68 \pm 1$ & $7 I \pm I$ & 0.168 \\
\hline Male, n (\%) & $61(62.9)$ & $40(57.1)$ & 0.522 \\
\hline \multicolumn{4}{|l|}{ Risk factors } \\
\hline Family history of CAD, n (\%) & $34(35.1)$ & $21(30.0)$ & 0.510 \\
\hline Smoking, n (\%) & $51(52.6)$ & $31(44.3)$ & 0.347 \\
\hline Hypertension, n (\%) & $74(76.3)$ & $58(82.9)$ & 0.340 \\
\hline Diabetes, n (\%) & $31(32.0)$ & $28(40.0)$ & 0.326 \\
\hline Hyperlipidemia, n (\%) & $73(75.3)$ & $47(67.1)$ & 0.296 \\
\hline Obesity, n (\%) & $33(34.0)$ & $23(32.9)$ & 1.000 \\
\hline Previous history of $\mathrm{PCI}, \mathrm{n}(\%)$ & $15(15.5)$ & $12(17.1)$ & 0.833 \\
\hline Previous history of CABG, $\mathrm{n}(\%)$ & $4(4.1)$ & $2(2.9)$ & 0.707 \\
\hline \multicolumn{4}{|l|}{ Medications } \\
\hline ACE inhibitors, $\mathrm{n}(\%)$ & $37(38.1)$ & $35(50.0)$ & 0.155 \\
\hline$\beta$-Blockers, n (\%) & $39(40.2)$ & $24(34.3)$ & 0.518 \\
\hline $\mathrm{CCB}, \mathrm{n}(\%)$ & $25(25.8)$ & $23(32.9)$ & 0.387 \\
\hline Statins, n(\%) & $49(50.5)$ & $38(54.3)$ & 0.641 \\
\hline $\mathrm{SBP}, \mathrm{mmHg}$ & $138 \pm 2$ & $138 \pm 2$ & 0.979 \\
\hline $\mathrm{DBP}, \mathrm{mmHg}$ & $80 \pm 1$ & $80 \pm 2$ & 0.944 \\
\hline Total cholesterol, $\mathrm{mmol} / \mathrm{L}$ & $\mid 72.1 \pm 4.7$ & $168.5 \pm 5.0$ & 0.512 \\
\hline Triglyceride, $\mathrm{mg} / \mathrm{dL}$ & | $49.8 \pm 9.1$ & $150.3 \pm 13.0$ & 0.973 \\
\hline $\mathrm{LDL}, \mathrm{mg} / \mathrm{dL}$ & $118.3 \pm 3.5$ & $121.4 \pm 4.0$ & 0.560 \\
\hline $\mathrm{HDL}, \mathrm{mg} / \mathrm{dL}$ & $44.4 \pm I .1$ & $44.3 \pm 1.5$ & 0.977 \\
\hline $\mathrm{HbA}_{\mathrm{IC}}, \mathrm{mmol} / \mathrm{L}$ & $6.6 \pm 0.1$ & $6.5 \pm 0.1$ & 0.593 \\
\hline Pre-PCl cTnl, $\mu g / L$ & $0.02 \pm 0.00$ & $0.05 \pm 0.01$ & 0.040 \\
\hline
\end{tabular}

Note: Data presented as mean \pm standard deviation unless stated otherwise. Abbreviations: $\mathrm{PCl}$, percutaneous coronary intervention; $\mathrm{PPCP}$, post- $\mathrm{PCl}$ chest pain; CAD, coronary artery disease; CABG, coronary artery bypass graft; $C C B$, calcium channel blocker; SBP, systolic blood pressure; DBP, diastolic blood pressure; LDL, low-density lipoprotein; HDL, high-density lipoprotein; cTnl, cardiac troponin I; $\mathrm{HbA}_{\mathrm{IC}}$, glycated hemoglobin.

groups. Further analyzing PPCP patients, 19 (27.1\%) had no ECG abnormality and cTnI elevation after PCI, eleven (15.7\%) had post-PCI cTnI elevation only, 20 (28.6\%) had abnormal post-PCI ECG only (including ST-segment depression, new Q-waves, or T-waves inversion), and 20 (28.6\%) had post-PCI cTnI elevation and abnormal ECG (Figure 2). These four subgroups showed no difference in clinical characteristics, PCI treatment, and short-term outcomes.

\section{Predictors of PPCP and post-PCI repeat revascularization}

As shown in Table 3, Pearson correlation test showed that the occurrence of PPCP in our cohort of patients with stable angina was correlated with abnormal post-PCI ECG changes such as new ST-segment depression and/or specific T-wave inversion $(P<0.0001)$, cTnI elevation $(P<0.0001)$, post-PCI cTnI level $(P<0.0001)$, number of stents placed $(P=0.009)$, and pre-PCI cTnI level $(P=0.049)$. Logistic regression analysis revealed post-PCI ECG changes $(P<0.0001)$, post-PCI cTnI level $(P<0.0001)$, and cTnI elevation $(P<0.0001)$ as 
Table $2 \mathrm{PCl}$ and short-term characteristics in the PPCP versus non-PPCP groups

\begin{tabular}{|c|c|c|c|}
\hline & Non-PPCP $(n=97)$ & $\operatorname{PPCP}(n=70)$ & $P$-value \\
\hline Intervention vessels, $\mathrm{n}(\%)$ & & & 0.038 \\
\hline I & $67(69.1)$ & $4 \mid(58.6)$ & - \\
\hline 2 & $29(29.9)$ & $23(32.9)$ & - \\
\hline 3 & I (I.0) & $6(8.5)$ & 0.020 \\
\hline Stent placement, n (\%) & & & 0.125 \\
\hline None & $19(19.6)$ & $8(11.4)$ & - \\
\hline Bare-metal stent & $46(47.4)$ & $35(50.0)$ & 0.756 \\
\hline Drug-eluting stent & $32(33.0)$ & $27(38.6)$ & 0.513 \\
\hline Number of stent placement & $1.2 \pm 0.1$ & $1.5 \pm 0.1$ & 0.007 \\
\hline Post-PCI ECG changes, n(\%) & & & $<0.0001$ \\
\hline Improved & $44(45.4)$ & $30(42.9)$ & 0.748 \\
\hline Unchanged & $53(54.6)$ & $20(28.6)$ & 0.0008 \\
\hline New Q-waves, ST-segment shifts, or T-waves inversion & 0 & $20(28.6)$ & $<0.0001$ \\
\hline Post-Tnl, $\mu g / L$ & $0.08 \pm 0.00$ & $\mathrm{I} .5 \mathrm{I} \pm 0.64$ & 0.007 \\
\hline Number of patients with post-PCl cTnl elevation, $\mathrm{n}$ (\%) & 0 & $31(44.3)$ & $<0.0001$ \\
\hline Number of patients with post-PCI MI, n (\%) & 0 & $2(2.9)$ & 0.174 \\
\hline Number of patients with repeat revascularization after $\mathrm{PCl}, \mathrm{n}(\%)$ & $9(9.3)$ & $15(21.4)$ & 0.043 \\
\hline Death, n (\%) & I (I.0) & $4(5.7)$ & 0.162 \\
\hline
\end{tabular}

Notes: Data presented as mean \pm standard deviation unless stated otherwise. “-” indicates not applicable.

Abbreviations: PCl, percutaneous coronary intervention; PPCP, post PCl chest pain; cTnl, cardiac troponin I; MI, myocardial infarction; ECG, electrocardiogram.

the strongest predictors of PPCP, followed by the number of stents placed $(P=0.048)$ (Table 4$)$.

Moreover, repeat revascularization after index PCI was significantly correlated to abnormal post-PCI ECG changes (new ST-segment depression and/or specific T-wave inversion) (Wald: $21.32, P<0.0001,95 \%$ confidence interval [CI]: 1.25-3.10).

\section{Discussion}

This study demonstrated that the incidence of PPCP occurring 24 hours after PCI was seen in $41.9 \%$ of our study cohort with stable angina. The PPCP patients are more likely to have abnormal ECG changes and cTnI elevation after PCI, more PCI vessels, and stent placement. Also, more PPCP patients required repeat revascularization than non-PPCP patients. The strongest predictor of PPCP was abnormal ECG changes

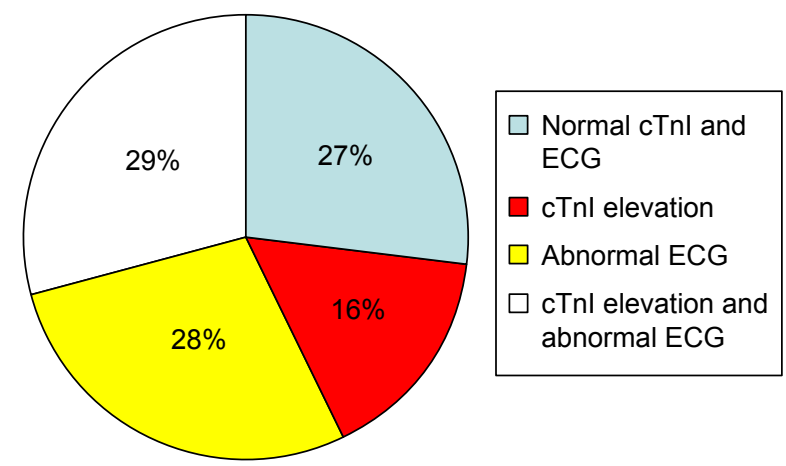

Figure 2 The proportion of patients with or without cTnl elevation and/or abnormal post-PCI ECG in 70 PPCP patients.

Abbreviations: $\mathrm{PCl}$, percutaneous coronary intervention; $\mathrm{PPCP}$, post- $\mathrm{PCl}$ chest pain; cTnl, cardiac troponin I; ECG, electrocardiogram. and cTnI elevation after PCI, followed by the number of stents placed. The rate of repeat revascularization after index PCI was correlated with abnormal post-PCI ECG changes.

According to our findings, more than half the PPCP patients had abnormal ECG changes after the PCI procedure. The incidence of PPCP was strongly associated with abnormal post-PCI ECG, indicating that PPCP may be caused by myocardial ischemia. However, caution should be taken when interpreting the findings about ECG changes. Without concomitant increase in serum cTnI, the most important changes in post-PCI ECG for myocardial ischemia in PCI patients are new ST-segment depression and/or specific $\mathrm{T}$-wave inversion. In patients with stable angina, chest pain can be caused by procedure-relevant factors or persistent symptoms of angina when PCI has actually failed. Previous studies have proven that PCI is effective in patients with medically refractory myocardial ischemia, ${ }^{16}$ but its advantage over medical therapy alone to improve the symptoms of angina in patients with chronic stable angina is limited. ${ }^{17,18}$

Table 3 Predictors of post- $\mathrm{PCl}$ chest pain in patients with stable angina

\begin{tabular}{lll}
\hline Predictors & $\begin{array}{l}\text { Correlation } \\
\text { coefficient }\end{array}$ & P-value \\
\hline $\begin{array}{l}\text { Abnormal changes in post-PCl } \\
\text { cTnl level }\end{array}$ & 0.620 & $<0.0001$ \\
Post-PCI ECG & 0.567 & $<0.000 I$ \\
cTnl elevation & 0.561 & $<0.000$ I \\
Number of stent placement & 0.268 & 0.009 \\
Pre-PCl cTnl level & 0.153 & 0.049 \\
\hline
\end{tabular}

Abbreviations: $\mathrm{PCl}$, percutaneous coronary intervention; $\mathrm{cTnl}$, cardiac troponin I; ECG, electrocardiogram. 
Table 4 Factors associated with post-PCl chest pain by logistic regression analysis

\begin{tabular}{llll}
\hline & Wald & P-value & $\begin{array}{l}95 \% \mathrm{Cl} \\
\text { (lower-upper) }\end{array}$ \\
\hline Abnormal changes in post-PCI ECG & 41.25 & $<0.000 I$ & $3.48-10.23$ \\
Tnl elevation & 23.09 & $<0.0001$ & $0.54-1.28$ \\
Post-PCl cTnl level & 6.42 & $<0.000$ I & $1.57-46.33$ \\
Number of stent placement & 3.74 & 0.048 & $0.93-2.44$ \\
\hline
\end{tabular}

Abbreviations: $\mathrm{PCl}$, percutaneous coronary intervention; cTnl, cardiac troponin I; $\mathrm{Cl}$, confidence interval; ECG, electrocardiogram.

In our cohort, 53 patients in the non-PPCP group had generally abnormal post-PCI ECG such as ST depression, but serum cTnI levels did not increase and chest pain was not much different from that before PCI. As we did not find any difference in other parameters associated with myocardial injury, we assume that the chest pain in these patients was caused predominantly by previous ischemic insult. In other words, the ineffectiveness of the PCI treatment cannot be ruled out in these patients. The presence of new ST-segment depression and/or T-wave inversion, even without cTnI elevation, seems more valuable to predict PPCP and repeat revascularization following PCI, which presented in $28.6 \%$ of PPCP patients but none of non-PPCP patients.

Another possible cause of PPCP in these patients is stent-stretch. Despite the fact that latest European Society of Cardiology guidelines only recommend PCI for severe ischemia or symptoms not being controlled by optimal medical treatment, ${ }^{19}$ the number of PCI cases in patients with chronic stable angina still continuously increases. ${ }^{7}$ This is mainly due to the evidence regarding the effectiveness of drug-eluting stents on reducing restenosis in both stable and unstable patients. ${ }^{20}$ Kini et a ${ }^{11}$ evaluated 176 patients with PPCP after coronary stenting and found significant elevation of CK-MB isoenzyme, larger postprocedure minimum lumen diameter, and higher stent-to-vessel ratio, suggesting micromyonecrosis and vessel stretch as causes of PPCP. Our findings also correlated the incidence of PPCP and stent placement, suggesting that PPCP may be due to stent-stretch in some patients. Furthermore, $27.1 \%$ of PPCP patients had normal ECG and cTnI level after PCI, suggesting that the possible cause of PPCP in these patients is likely to be stent-stretch.

After successful PCI, cTnI elevation is not rare. ${ }^{21-25}$ Studies have shown that cTnI elevation is closely associated with myocardial injury, and $\mathrm{cTnI}$ is the most sensitive marker for the detection of major complications in patients undergoing PCI. ${ }^{26-28}$ However, these studies only evaluate the predictive value cTnI elevation on myocardial injury and prognosis. The relationship between PPCP and cTnI elevation is poorly investigated. In a cohort of 199 patients who underwent PCI for stable and unstable angina, Schüepp et $\mathrm{a}^{10}$ found that the incidence of TnI elevation was $29.8 \%$ within 16 hours after PCI. Significantly more patients with chest pain had $\mathrm{TnI}$ levels more than twice the upper normal limit than patients without chest pain. Okmen et al ${ }^{29}$ also reported that cTnI elevation was detected in $34 \%$ of patients with angina. Patients with stable angina were more likely to have less cTnI elevation than those with unstable angina. The association between cTnI elevation and stent placement after balloon dilation with maximal inflation pressure was only found in patients with unstable angina. In the present study, we found that 31 of $167(18.6 \%)$ patients with stable angina had cTnI elevation after PCI, which is less than that observed in aforementioned studies. But cTnI elevation is significantly correlated to the incidence of PPCP. The underlying mechanisms remain obscure. One possible mechanism is ischemic damage, as two patients diagnosed with post-PCI MI had more than 50 times the upper normal limit of serum cTnI level. Another possible mechanism may be related to endothelial damage because of stent implantation, indicated by more numbers of stents placed in the PPCP group. Statistical analysis also detects a significant correlation between the two. Further large-scale studies are needed to confirm these findings.

\section{Limitations}

The current study has some limitations. First, it is a singlecenter retrospective study, and certain proportion of patients without complete data about ECG, cTnI, and PCI details were excluded. Therefore, some bias may exist and limit the generalization of the results. For example, the incidence of PPCP is approximately $42 \%$, which is higher than $30 \%-35 \%$ in previous reports. Second, more than $80 \%$ of patients had stent implantation in our cohort, which is much higher than the practice in Western countries. This reflects the health care condition in the People's Republic of China, although the benefit of stent placement in reducing the risk of cardiovascular events has not been confirmed in patients with stable angina. Our results did not show better outcomes with stent placement, but we do find that the number of stents placed is correlated to the incidence of PPCP. A multicenter prospective study is needed.

\section{Conclusion}

In conclusion, PPCP occurring 24 hours after PCI may be common in patients with stable angina. PPCP is associated with stent placement and can be predicted by abnormal ECG and elevated serum cTnI level. 


\section{Disclosure}

The authors report no conflicts of interest in this work.

\section{References}

1. Parisi AF, Folland ED, Hartigan P; Veterans Affairs ACME Investigators. A comparison of angioplasty with medical therapy in the treatment of single-vessel coronary artery disease. $N$ Engl J Med. 1992;326: $10-16$.

2. Myler RK, Shaw RE, Stertzer SH, et al. Unstable angina and coronary angioplasty. Circulation. 1990;82:II88-II95.

3. Grines CL, Browne KF, Marco J, et al; The Primary Angioplasty in Myocardial Infarction Study Group. A comparison of immediate angioplasty with thrombolytic therapy for acute myocardial infarction. N Engl J Med. 1993;328:673-679.

4. Society BCI. Percutaneous Coronary Interventions Annual Public Report. London, UK: NICOR, 2013. Available from: https://www.ucl. ac.uk/nicor/audits/adultpercutaneous/documents/2013_annual_report_ pdf. Accessed April 26, 2016.

5. Gorenoi V, Schonermark MP, Hagen A. Percutaneous coronary intervention with optimal medical therapy vs. optimal medical therapy alone for patients with stable angina pectoris. GMS Health Technol Assess. 2011; 7:Doc07.

6. Kozinski M, Sukiennik A. Revisiting the role of percutaneous coronary interventions in stable angina: the landscape after the COURAGE trial. Cardiol J. 2007;14:321-325.

7. Maragiannis D, Lazaros G, Vavuranakis M, et al. Chronic stable angina: percutaneous coronary intervention or medication? Hellenic J Cardiol. 2011;52:246-252.

8. Kozuch M, Kralisz P, Korecki J, et al. Early and long-term prognosis of patients with coronary artery disease treated with percutaneous coronary interventions in 2005. Experience of single large-volume PCI center. Adv Med Sci. 2011;56:222-230.

9. Tebbe U, Hochadel M, Bramlage P, et al. In-hospital outcomes after elective and non-elective percutaneous coronary interventions in hospitals with and without on-site cardiac surgery backup. Clin Res Cardiol. 2009; 98:701-707.

10. Schüepp M, Ullmer E, Weinbacher M, et al. Chest pain early after percutaneous coronary intervention: incidence and relation to ECG changes, cardiac enzymes and follow-up events. J Invasive Cardiol. 2001;13: 211-216.

11. Kini AS, Lee P, Mitre CA, Duffy ME, Sharma SK. Postprocedure chest pain after coronary stenting: implications on clinical restenosis. $J$ Am Coll Cardiol. 2003;41:33-38.

12. Jeremias A, Kutscher S, Haude M, et al. Nonischemic chest pain induced by coronary interventions: a prospective study comparing coronary angioplasty and stent implantation. Circulation. 1998;98:2656-2658.

13. Mansour M, Carrozza JP Jr, Kuntz RE, et al. Frequency and outcome of chest pain after two new coronary interventions (atherectomy and stenting). Am J Cardiol. 1992;69:1379-1382.

14. Kini A, Marmur JD, Kini S, et al. Creatine kinase-MB elevation after coronary intervention correlates with diffuse atherosclerosis, and lowto-medium level elevation has a benign clinical course: implications for early discharge after coronary intervention. J Am Coll Cardiol. 1999;34: 663-671.
15. Smith SC Jr, Feldman TE, Hirshfeld JW Jr, et al. ACC/AHA/SCAI 2005 guideline update for percutaneous coronary intervention: a report of the American College of Cardiology/American Heart Association Task Force on Practice Guidelines (ACC/AHA/SCAI Writing Committee to Update 2001 Guidelines for Percutaneous Coronary Intervention). Circulation. 2006;113:e166-e286.

16. Stroupe KT, Morrison DA, Hlatky MA, et al. Cost-effectiveness of coronary artery bypass grafts versus percutaneous coronary intervention for revascularization of high-risk patients. Circulation. 2006;114: $1251-1257$

17. Coronary angioplasty versus medical therapy for angina: the second Randomised Intervention Treatment of Angina (RITA-2) trial. RITA-2 trial participants. Lancet. 1997;350:461-468.

18. Sculpher M, Smith D, Clayton T, et al. Coronary angioplasty versus medical therapy for angina. Health service costs based on the second Randomized Intervention Treatment of Angina (RITA-2) trial. Eur Heart J. 2002;23:1291-1300.

19. Wijns W, Kolh P, Danchin N, et al. Guidelines on myocardial revascularization. Eur Heart J. 2010;31:2501-2555.

20. Ramsdale DR, Rao A, Asghar O, Ramsdale KA, McKay E. Late outcomes after drug-eluting stent implantation in "real-world" clinical practice. J Invasive Cardiol. 2008;20:493-500.

21. Garbarz E, Iung B, Lefevre G, et al. Frequency and prognostic value of cardiac troponin I elevation after coronary stenting. Am J Cardiol. 1999; 84:515-518.

22. La Vecchia L, Bedogni F, Finocchi G, et al. Troponin T, troponin I and creatine kinase-MB mass after elective coronary stenting. Coron Artery Dis. 1996;7:535-540.

23. Cantor WJ, Newby LK, Christenson RH, et al. Prognostic significance of elevated troponin I after percutaneous coronary intervention. $J \mathrm{Am}$ Coll Cardiol. 2002;39:1738-1744.

24. Tricoci P, Leonardi S, White J, et al. Cardiac troponin after percutaneous coronary intervention and 1-year mortality in non-ST-segment elevation acute coronary syndrome using systematic evaluation of biomarker trends. J Am Coll Cardiol. 2013;62:242-251.

25. Selvanayagam JB, Porto I, Channon K, et al. Troponin elevation after percutaneous coronary intervention directly represents the extent of irreversible myocardial injury: insights from cardiovascular magnetic resonance imaging. Circulation. 2005;111:1027-1032.

26. Miller WL, Garratt KN, Burritt MF, Reeder GS, Jaffe AS. Timing of peak troponin $\mathrm{T}$ and creatine kinase-MB elevations after percutaneous coronary intervention. Chest. 2004;125:275-280.

27. Bolognese L, Ducci K, Angioli P, et al. Elevations in troponin I after percutaneous coronary interventions are associated with abnormal tissuelevel perfusion in high-risk patients with non-ST-segment-elevation acute coronary syndromes. Circulation. 2004;110:1592-1597.

28. Wong GC, Morrow DA, Murphy S, et al; Treat Angina with Aggrastat and Determine Cost of Therapy with an Invasive or Conservative Strategy-Thrombolysis in Myocardial Infarction. Elevations in troponin $\mathrm{T}$ and I are associated with abnormal tissue level perfusion: a TACTICSTIMI 18 substudy. Circulation. 2002;106:202-207.

29. Okmen E, Cam N, Sanli A, Unal S, Tartan Z, Vural M. Cardiac troponin I increase after successful percutaneous coronary angioplasty: predictors and long-term prognostic value. Angiology. 2006;57: 161-169.
Clinical Interventions in Aging

\section{Publish your work in this journal}

Clinical Interventions in Aging is an international, peer-reviewed journal focusing on evidence-based reports on the value or lack thereof of treatments intended to prevent or delay the onset of maladaptive correlates of aging in human beings. This journal is indexed on PubMed Central, MedLine,

\section{Dovepress}

CAS, Scopus and the Elsevier Bibliographic databases. The manuscript management system is completely online and includes a very quick and fair peer-review system, which is all easy to use. Visit http://www.dovepress. com/testimonials.php to read real quotes from published authors. 